\title{
Role of Telemedicine in Follow-up Care of Children with Respiratory Illnesses at a Tertiary Care Hospital - An Ambispective Observational Study
}

\author{
Jyoti Kumari ${ }^{1} \cdot$ Kana Ram Jat $^{1}$ (D) - S. K. Kabra ${ }^{1}$ \\ Received: 26 September 2020 / Accepted: 25 November 2020 / Published online: 4 January 2021 \\ (C) Dr. K C Chaudhuri Foundation 2021
}

\begin{abstract}
Objective To assess if telemedicine can be used successfully for follow-up care of children with respiratory illnesses. The authors also assessed problems faced by the doctors and satisfaction of caregivers of these patients with telemedicine.

Methods The authors conducted an ambispective observational study. Data related to demographic details and diagnoses of patients who had telemedicine consultation (teleconsultation) appointments between 2nd April 2020 to 15th May 2020 were reviewed retrospectively. They noted proportion of patients having successful prescription. To assess problems faced by doctors and satisfaction of caregiver of patients with teleconsultation, a prospective questionnaire was sent via Google Forms 6-10 wk after the initial appointment date. Those who did not respond to Google Forms were called by phone to assess the same.

Results A total of 188 patients received teleconsultation during the study period. Team was able to prescribe treatment in 181 (96.3\%) patients via teleconsultation and other seven (3.7\%) required physical evaluation. Mean (SD) age of patients was 9.7 (4.9) y, range 3 mo to $18 \mathrm{y}$. There were $117(62.2 \%)$ boys and $71(37.8 \%)$ girls. Majority (58\%) of the patients were asthmatics. The team advised refill prescription in $83 \%$ patients as symptoms were controlled. Three out of five residents faced minor problems while providing teleconsultation. In satisfaction assessment, $78 \%$ of caregivers rated teleconsultation 8 or more, out of 10 points, suggesting that most of them were satisfied with telemedicine.

Conclusion In majority of children with respiratory illnesses, successful follow-up care can be provided by telemedicine.
\end{abstract}

Keywords Children $\cdot$ COVID-19 $\cdot$ Respiratory $\cdot$ Telemedicine

\section{Introduction}

Corona virus disease (COVID-19) pandemic and nationwide lockdown has led to the disruption of healthcare services. The majority of hospitals have shut down routine outpatient departments and follow-up clinics with only emergency services and fever/flu clinics running. Telemedicine has a pivotal role in such scenarios. Telemedicine provides an easily accessible, cost-effective, and timely health care service. World Health

Supplementary Information The online version contains supplementary material available at https://doi.org/10.1007/s12098-02003590-8.

Kana Ram Jat

drkanaram@gmail.com

1 Department of Pediatrics, All India Institute of Medical Sciences, New Delhi 110029, India
Organization defines telemedicine as "the delivery of health care services, where distance is a critical factor, by all health care professionals using information and communication technologies for the exchange of valid information for diagnosis, treatment and prevention of disease and injuries, research and evaluation, and for the continuing education of health care providers, all in the interests of advancing the health of individuals and their communities" [1]. In simple words, telemedicine means providing medical care at a site distant to the healthcare provider using technology. Until recently, telemedicine was used sparsely in India as there were no proper guidelines. The recent launch of Telemedicine Practice Guidelines by the Medical Council of India (MCI) and the ongoing COVID-19 pandemic has led to an increase in telemedicine services across the country and doctors are more confident in providing such services [2]. To contain spread of SARS-CoV2 , it is recommended to prefer telemedicine services wherever feasible [3]. Telemedicine has been used for patient care in various specialities, including management of obesity, 
diabetes, epilepsy and psychological disorders in adults as well as children and has been shown to be cost-effective [4-7]. However, there is hardly any study assessing the utility of telemedicine for follow-up care of children with respiratory illnesses. The authors did an ambispective observational study to see whether telemedicine can be used successfully for follow-up care of children with respiratory illnesses. They also assessed the satisfaction of caregivers of these patients with telemedicine services and the problems faced by the doctors providing these services.

\section{Material and Methods}

This was an ambispective observational study conducted in the Department of Pediatrics at a tertiary care institute in North India. Ethical clearance was taken from Institute Ethics Committee. Teleconsultation data of all patients aged less than 18 y who had an appointment from 2nd April 2020 to 15th May 2020 were reviewed retrospectively. The procedure followed at the Department of Pediatrics at the authors' institute is as follows: (1) patient takes appointment online via website, app, or phone call; (2) the computer facility sends the list of appointments to the department; and (3) the teleconsultation team of the department calls the patients on the day of appointment for teleconsultation and after taking consent, assesses the details of the child's condition and provides suggestions/prescription. All teleconsultations were provided by post-MD residents in consultation with faculty as and when required. Data of patients who had appointments but could not be contacted due to various reasons (wrong number, phone not reachable, phone switched off, did not pick up the call) were excluded. All teleconsultations were synchronous, audio-based (aided by text-message and WhatsApp media), between doctor and caregiver. For this study, we retrieved demographic data (age, sex), and diagnosis of primary disease of the patients. Primary objective of the study was to assess what proportion of patients could be successfully prescribed by telemedicine. Secondary objectives of the study were: (1) to prospectively assess satisfaction of caregivers of these patients with telemedicine services; and (2) to assess problems faced by the doctors providing telemedicine services. Satisfaction of the caregivers of these patients was assessed by sending a small questionnaire in English and Hindi via Google Forms about 6-10 wk after teleconsultation (Supplementary Table 1a and b). Questionnaire was devised considering common concerns of caregivers and doctors and inputs from resident doctors who provided teleconsultation. Questionnaire was translated to Hindi using Google Translate and then edited into easy-to-understand language.

The satisfaction level was assessed on a scale of 10 points; 10 being most satisfied. Problems faced by the doctors were also assessed prospectively by sending them a small questionnaire via google form (Supplementary Table 1c). If the authors did not get a response or if the response was incomplete via Google Forms, they called the attendants/ caregivers to ask the same questions. Data were entered on the excel sheet and analyzed using Microsoft Excel.

\section{Results}

During the study period, a total of 247 patients had appointments out of which, 188 could be contacted for teleconsultation, while 59 could not be contacted because of various reasons (wrong number, phone not reachable, switched off, did not pick up the call). Team was able to prescribe in $181(96.3 \%)$ patients and seven (3.7\%) required physical evaluation. Mean (SD) age of patients was 9.7 (4.9) $\mathrm{y}$, ranging from $3 \mathrm{mo}$ to $18 \mathrm{y}$. There were 117 (62.2\%) boys and $71(37.8 \%)$ girls. Majority $(58 \%)$ of the patients were asthmatics. Tables 1 and 2 show diagnoses of patients who received telemedicine services and advice given to them via teleconsultation respectively. Majority $(83.5 \%)$ of the patients were continued on same treatment (refill prescription) as symptoms of underlying disease were controlled.

\section{Assessment of Satisfaction of Caregiver with Teleconsultations}

Response was received from $121(64.4 \%)$ caregivers; 42 via Google Forms and 79 via phone calls. The respondents were father in $60 \%$, mother in $30 \%$, while $10 \%$ were other relatives of patient. Most of the respondents remember the teleconsultation very well. Rating of 8 or more, out of 10 points, was provided by $93(78.1 \%)$ respondents for telemedicine services and rating of 10 by $56(47.1 \%)$. Only eight (6.6\%) respondents rated below 5 .

A total of 95 (78.5\%) respondents wanted to continue with teleconsultation rather than in-person consultation. However,

Table 1 Diagnoses of patients who received telemedicine services during the study period $(N=188)$

\begin{tabular}{lll}
\hline S. no. & Diagnosis & Number of patients, n (\%) \\
\hline 1 & Asthma & $109(58 \%)$ \\
2 & Primary ciliary dyskinesia & $19(10.1 \%)$ \\
3 & Active tuberculosis & $19(10.1 \%)$ \\
4 & Cystic fibrosis (CF) & $11(5.8 \%)$ \\
5 & Interstitial lung disease & $10(5.3 \%)$ \\
6 & Treated tuberculosis $^{\text {a }}$ & $8(4.3 \%)$ \\
7 & Others $^{\text {a }}$ & $12(6.4 \%)$ \\
\hline
\end{tabular}

${ }^{a}$ Others included aspiration pneumonia, lung malformation, non-CF bronchiectasis, bronchopulmonary dysplasia 
Table 2 Advice given to patients on teleconsultation $(\mathrm{N}=188)$

\begin{tabular}{lll}
\hline S. no. & Advice given & $\begin{array}{l}\text { No. of patients, } \mathrm{n}(\%) \\
\text { Total }=188\end{array}$ \\
\hline 1 & Continue same treatment (refill prescription) & $157(83.5 \%)$ \\
2 & Step-up/step-down treatment for underlying disease & $16(8.5 \%)$ \\
3 & Treatment for new complaint (not related to underlying disease) & $8(4.2 \%)$ \\
4 & Visit local physician (urgent) & $5(2.7 \%)$ \\
5 & Visit our hospital (nonurgent) & $4(2.1 \%)$
\end{tabular}

${ }^{\mathrm{a}}$ Two patients were advised treatment for new complaint (not related to underlying disease) along with visit to local physician only 48 (40\%) of them wanted to continue it after the COVID19 pandemic is over. One hundred and five (87\%) would recommend teleconsultation to others. Fifty (45\%) respondents wanted to continue with audio consultation while 61 (55\%) wanted video consultation next time.

Figure 1 shows problems faced by the caregivers. Most of the caregivers $(69.6 \%)$ did not face any problem while availing teleconsultations. Around $83 \%$ of caregivers wanted to continue telemedicine because of ongoing COVID-19 pandemic, while $27 \%$ found it similar to in-person consultation. Figure 2 shows reasons for continuing telemedicine as told by caregivers of the patients. About 13\% did not want to continue telemedicine services.

\section{Problems Faced by the Doctors}

Six post-MD residents provided teleconsultation during the study period. Five $(83.3 \%)$ responded to Google Forms. Three out of five residents faced a few problems while providing consultation via telemedicine. One resident found difficulty in explaining advice to patient/ caregiver on phone. Only one resident preferred teleconsultation over in-person visit. Two residents preferred video consultation over audio consultation for all consultations, while three would prefer only for selected cases. Video consultation was suggested by most for next consultation.

\section{Discussion}

The present study suggests that vast majority of children with respiratory illnesses who are under follow-up care can be successfully managed via telemedicine. Around $80 \%$ of the caregivers were satisfied with telemedicine services and wanted to continue it, however only half of them wanted to continue telemedicine after COVID-19 pandemic is over. Most of them did not face any problem while consulting via telemedicine. Most residents and caregivers suggested video consultation over audio consultation.

There are very few studies assessing role of telemedicine in follow-up care of children with respiratory illnesses. In a multicenter, randomized controlled trial by Wijngaart et al., 210 asthmatic children were randomized to receive either usual care (4-monthly outpatient visits) or online care using virtual
Fig. 1 Problems faced by the caregivers with teleconsultation (112 responded to this question). Others included inability to show reports

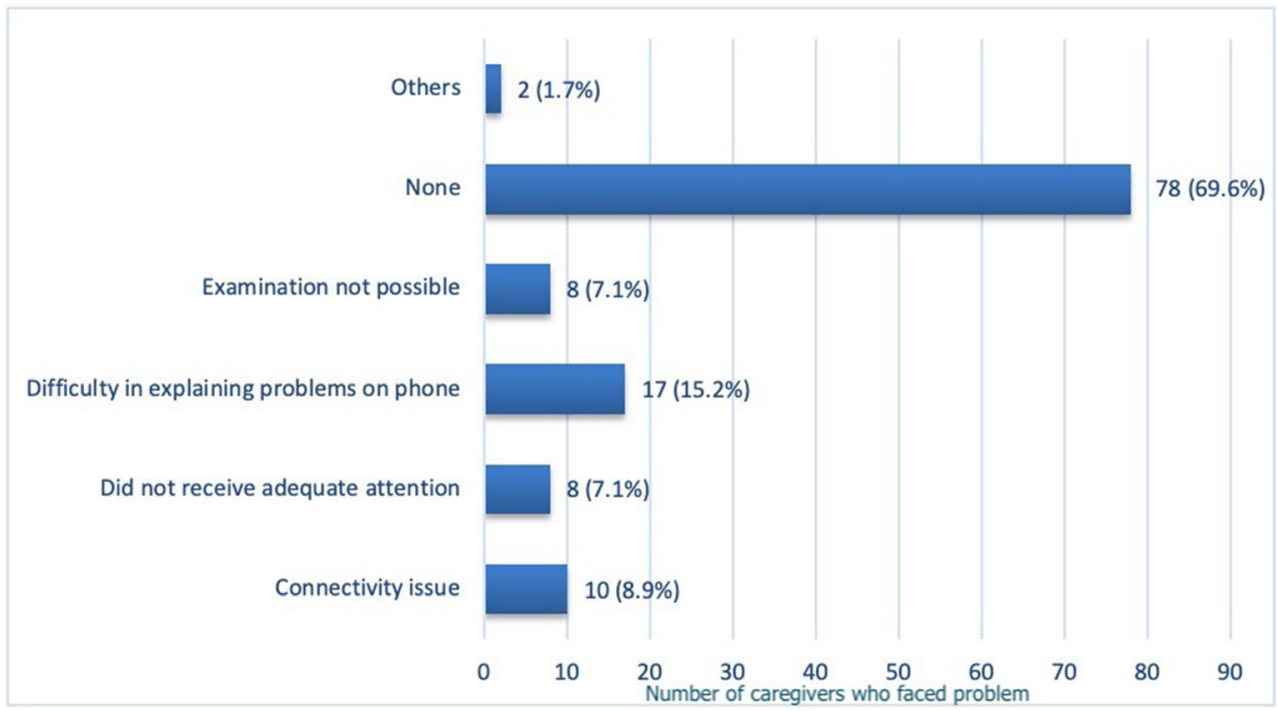


Fig. 2 Reason for continuing teleconsultation as told by caregiver (119 responded to this question)

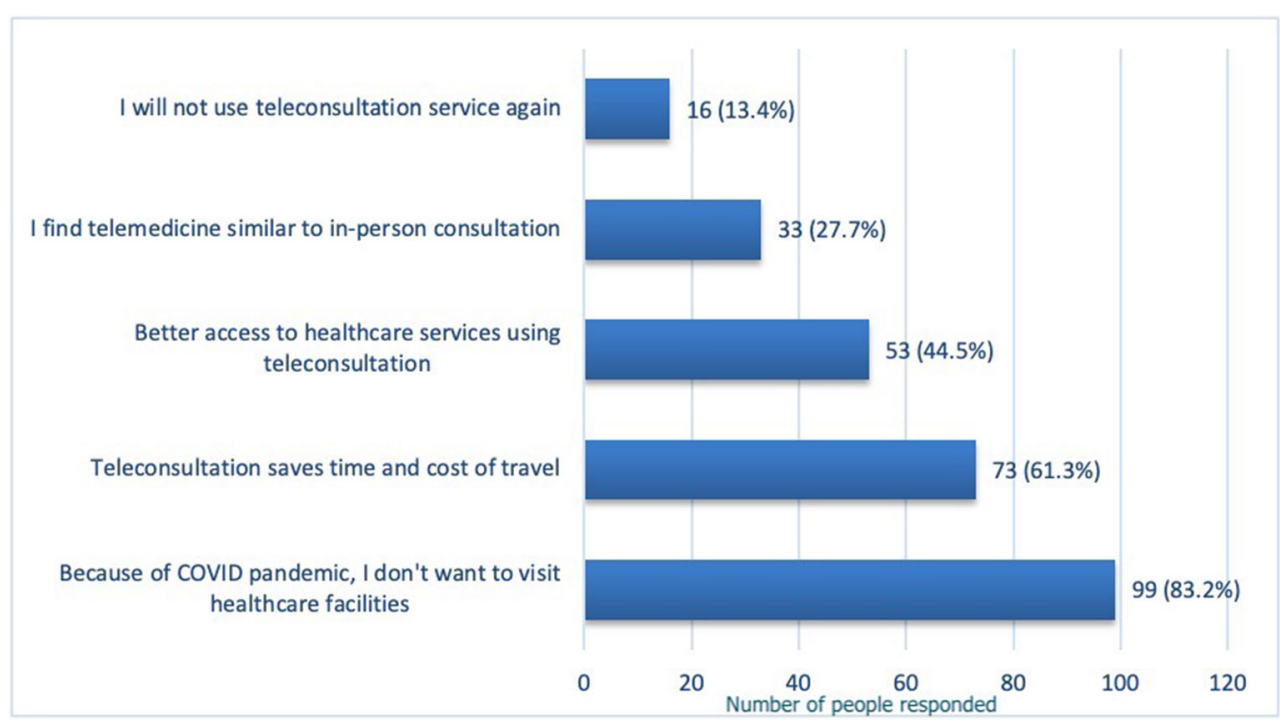

asthma clinic (VAC) (8-monthly outpatient visits with monthly web-based monitoring). Asthmatic children receiving VAC had significantly more symptom free days and better asthma control as compared to those receiving usual care [8]. VAC reduced outpatient visit by $50 \%$ and this did not affect the quality of life (assessed using questionnaires at 0,8 and 16 mo). Both direct and indirect costs per patient were significantly lower in the VAC group than in the usual care group mainly because of fewer outpatient visits and less travel expenses. Their study showed that online asthma management is both clinically effective and cost-effective and can partly replace outpatient visits [9]. Study by McKissick et al. showed that telehealth intervention delivered by advanced practice registered nurse to children with medical complexity led to reduction in number of unplanned health care visits (due to acute deterioration in child's condition) and increase in planned visit (for regular health maintenance) [10].

Apart from being cost-effective, telemedicine can also alleviate health care access issues, especially in rural areas [11, 12]. A survey conducted by Marcin et al. on children with special health care needs (CSHCN) living in a rural, medically underserved community found that overall satisfaction of parents/guardians and health care provider with telemedicine was very high, with most reporting it as "very good" or "excellent". Telemedicine can reduce time and cost of travel, work absenteeism for appointment and emergency department visits [13]. A systematic review by Lake et al. showed that patient satisfaction with telephone triage and advice services (TTAS) was comparable to usual care and it also reduced clinical workload. The study also compared the safety of TTAS with usual care (frequency of adverse events, errors and hospitalization rates) and found that TTAS was safe in most cases [14]. Recent systematic review by Orlando et al. also suggested that most of the patients and caregivers were satisfied with telemedicine [15]. In the present study also, the authors found that around $80 \%$ of caregivers rated telemedicine 8 or more out of 10 , suggesting that most of them were satisfied with telemedicine services. The main reason for continuing teleconsultation was to avoid visiting healthcare facility during the COVID-19 pandemic and that it saved time and cost of travel to hospital. In the current, COVID-19 pandemic, telemedicine services will definitely change healthcare delivery mode as many doctors are busy catering to COVID-19 patients. Also, as it reduces overcrowding in hospitals, it reduces risk of infection to patients, their attendants and healthcare workers. Hence, many authorities are recommending to enhance telemedicine services [3].

Strengths of the study: This is one of the few studies to assess the success of telemedicine in follow-up care of children with respiratory illnesses. The study also assessed the satisfaction of caregivers of these patients with telemedicine and the problems faced by them and by the doctors.

Limitations of the study: As majority of doctors and caregivers preferred video-consultation, satisfaction level could have been better with video consultation. Also, many caregivers opted for teleconsultation due to pandemic rather than actually preferring it over in-person consultation. The study included only follow-up cases, not the new cases with respiratory illness. Finally, there was no formal training of residents for tele-medicine, though were briefed about it and they consulted faculty in case of any problem.

Implication of study: During ongoing COVID-19 pandemic, the teleconsultation may be very useful in follow-up of children with respiratory illnesses. In resource limited setting, rural areas and during pandemics, telemedicine plays a vital role in delivery of healthcare services. Growing evidence suggests that both patients/ caregivers and health care providers are satisfied with telemedicine. 
Need for future studies: Future studies should assess the role of telemedicine in new patients with respiratory illness that should also include video-consultation.

\section{Conclusion}

In majority of children with respiratory illnesses, successful follow-up care can be provided by telemedicine.

Authors' Contributions SKK, KRJ has concept of idea; JK collected data and wrote initial manuscript; SKK, KRJ revised manuscript intellectually; all authors approved the final version of manuscript. KRJ will act as guarantor for this paper.

\section{Compliance with Ethical Standards}

Conflict of Interest None.

\section{References}

1. A health telematics policy in support of WHO's Health-for-all strategy for global health development : report of the WHO Group Consultation on Health Telematics, 11-16 December, Geneva, 1997. Available at: https://apps.who.int/iris/handle/10665/63857. Accessed 23 June 2020.

2. Telemedicine Practice Guidelines. Board of Governors in supersession of the Medical Council of India. 2020. Available at: https:// www.mohfw.gov.in/pdf/Telemedicine.pdf. Accessed 23 June 2020.

3. Infection Control Guidance for Healthcare Professionals about Coronavirus (COVID-19). Centers for Disease Control and Prevention. 2020. Available at: https://www.cdc.gov/coronavirus/ 2019-ncov/hcp/infection-control.html. Accessed 23 June 2020.

4. Hammersley ML, Jones RA, Okely AD. Parent-focused childhood and adolescent overweight and obesity ehealth interventions: a systematic review and meta-analysis. J Med Internet Res. 2016;18(7):e203.

5. Ahmed SN, Mann C, Sinclair DB, et al. Feasibility of epilepsy follow-up care through telemedicine: a pilot study on the patient's perspective. Epilepsia. 2008;49(4):573-85.

6. García-Lizana F, Muñoz-Mayorga I. Telemedicine for depression: a systematic review. Perspect Psychiatr Care. 2010;46(2):119-26.

7. Lee JY, Lee SWH. Telemedicine cost-effectiveness for diabetes management: a systematic review. Diabetes Technol Ther. 2018;20(7):492-500.

8. van den Wijngaart LS, Roukema J, Boehmer ALM, et al. A virtual asthma clinic for children: fewer routine outpatient visits, same asthma control. Eur Respir J. 2017;50:1700471.

9. van den Wijngaart LS, Kievit W, Roukema J, et al. Online asthma management for children is cost-effective. Eur Respir J. 2017;50: 1701413.

10. McKissick HD, Cady RG, Looman WS, Finkelstein SM. The impact of telehealth and care coordination on the number and type of clinical visits for children with medical complexity. J Pediatr Health Care. 2017;31(4):452-8.

11. Marcin JP, Shaikh U, Steinhorn RH. Addressing health disparities in rural communities using telehealth. Pediatr Res. 2016;79(1-2): 169-76.

12. Estrada RD, Ownby DR. Rural asthma: current understanding of prevalence, patterns, and interventions for children and adolescents. Curr Allergy Asthma Rep. 2017;17(6):37.

13. Marcin JP, Ellis J, Mawis R, Nagrampa E, Nesbitt TS, Dimand RJ. Using telemedicine to provide pediatric subspecialty care to children with special health care needs in an underserved rural community. Pediatrics. 2004;113(1 Pt 1):1-6.

14. Lake R, Georgiou A, Li J, et al. The quality, safety and governance of telephone triage and advice services - an overview of evidence from systematic reviews. BMC Health Serv Res. 2017;17:614.

15. Orlando JF, Beard M, Kumar S. Systematic review of patient and caregivers' satisfaction with telehealth videoconferencing as a mode of service delivery in managing patients' health. PLoS One. 2019;14(8):e0221848.

Publisher's Note Springer Nature remains neutral with regard to jurisdictional claims in published maps and institutional affiliations. 